\title{
A REVIEW ON RASAUSHADHIS MENTIONED IN BHAISHAJYA RATNAVALI FOR THE MANAGEMENT OF GRAHANI ROGA
}

\author{
DR. RADHIKA S. ${ }^{1}$, DR. V.S. SHREESHANANDA SHARMA ${ }^{2}$ \& DR. RAJENDRA PRASAD M.L. ${ }^{3}$ \\ ${ }^{1} P G$ Scholar, Department of Rasashastra and Bhaishajya Kalpana, JSSAMC, Mysuru \\ ${ }^{2}$ Professor \& HOD, Department of Rasashastra and Bhaishajya Kalpana, JSSAMC, Mysuru \\ 3. Assistant Professor, Department of Rasashastra and Bhaishajya Kalpana, JSSAMC, Mysuru
}

\begin{abstract}
Gastro-Intestinal(GIT) Disorders form a significant part of medical practice in the world. Since ancient times till date, Grahani Roga has been a precursor and is also a major source of many GIT ailments. The word Grahani indicates the seat of Agni (digestive fire) helping in the digestion and metabolism of food. In classics, Grahani has been described to perform the functions like ingestion, digestion, absorption and assimilation of Aahara. The faulty lifestyle like changed eating habits and their patterns, busy schedules with abnormal-stressful life events, inadequate sleep and nonobservance of Dinacharya, Ritucharya, Ratricharya and Sadvritta have been the etiological factors for thriving metabolic diseases within the community. Due to improper digestion of food, there is vitiation of the Doshas and Agni with associated predominant symptom being altered bowel habits like Constipation and Diarrhea, comprehensively termed as Grahani Roga. Symptomatology and causative factors of Grahani Roga can be considered similar to that of Irritable Bowel Syndrome. There are lots of classical texts available in Ayurveda but Bhaishajya Ratnavali by Govind Das Sen, a classic compendium contains a compilation and systematic categorization of the different herbal and herbomineral formulations \& Pathya-Apathya for each disease. Rasaushadhis are appraised for their instant relief at minuscule doses which are useful in treating the majority of grave disease conditions. In this article, an attempt is made to collect the details of Rasaushadhis used in Grahani Roga and to analyze the mode of action of these individual Bhasmas and formulations based on their ingredients and efficacy at the level of Dosha, Dushya, Samprapti and Lakshanas.
\end{abstract}

KEYWORDS: Grahani Roga, Irritable Bowel Syndrome, Rasaushadhi, Bhaishajya Ratnavali

Received: Jul 28, 2021; Accepted: Aug 18, 2021; Published: Aug 31, 2021; Paper Id.: IJMPSDEC20218

\section{INTRODUCTION}

Grahani has both anatomical and physiological significance in Ayurvedic science. In its physiological sense, it is closely associated with Agni, the metabolic principle. Grahani Roga is rife among the folk accustomed to insalubrious habits and suffering from nutritional paucity. The inappropriate way of life as in unwholesome food consumption, stress, sleep deprivation and non-observance of Sadvritta are the prime inducers of Grahani Roga. The disease is portrayed as having symptoms of alternating constipation and diarrhea which arise as a result of Ama formed due to vitiated Agni and Doshas, a consequence of anomalous digestion of food ${ }^{1-4}$. Irritable Bowel Syndrome (IBS) is a functional bowel disorder characterized by abdominal pain or discomfort and altered bowel habits in the absence of detectable structural abnormalities ${ }^{5}$.

Jatharagni is paramount to all living beings ${ }^{6}$. Adequate and timely intake of food in the equilibrium state of Agni results in proper digestion which would favor the maintenance of normalcy of Dhatu. Slackened digestive 
power leads to Vidaha (a part of food which is digested the other part remaining without digestion) either moving upwards or downwards in Mahastrotas and when the movement is downwards, it is termed as Grahani Roga. In this condition, the food stays in the Vidagdha Avastha giving rise to the symptoms like diarrhea or constipation, thirst, difficulty swallowing, tastelessness, excessive salivation, shortness of breath, fever, vomiting, and eructations ${ }^{7}$. In order to enhance the Agni for achieving eupepsia and to pacify Vata-Pitta Doshas, drugs having Kashaya Rasa, Ushna Veerya, Madhura Vipaka \& Ruksha Guna are used. Medications which offer mass to the stool, hydrate the body and have nutritional advantages reduce the symptoms of Grahani Roga.

\section{Grahani Roga /Dosha}

As per Acharya Charaka, Grahani is located above Nabhi (umbilical region). Agni resides in Grahani and reinforces it. It is named so because of its capacity to hold (Grahanat) food and restrain downward movement of undigested food and releases the digested food through its lumen. In the abnormal condition, when it gets vitiated because of weakness of Agni, it releases the food in undigested form itself ${ }^{8}$. Acharaya Sushruta describes Grahani as Shasti Pittadhara Kala located between Amashaya and Pakwashaya9. Ashtanga Sangraha quotes that Grahani does the functions like ingesting, digesting, absorbing and assimilating the Chaturvidha Ahara and ensuring the removal of waste product ${ }^{10}$. Chakrapani states that Grahani Dosha as Trividha abnormalities of Jatharagni ${ }^{11}$. Grahani Roga/Dosha has undoubtedly turned out to be one of the initiating factors of other illnesses. As indicated by Charaka, Agni being consequently vitiated, the individual doesn't even assimilate the lightest of meals. This indigestible food becomes acidulous to taste and becomes incompatible with the body $^{12}$. Acharya Sushruta says that a person tends to land in Grahani Dosha/Roga due to his misdemeanor of food and way of living after getting treated for Atisara ${ }^{13}$.

\section{Etiological Factors ${ }^{14}$}

$\square$ Abhojanat, Ajeernabhojanat, Attibhojanaat, Visamasanat, Asatmya Guru, Ruksa and Sandusta Bhojanat etc.
$\square$ Vyadhikarshanat and Vegavidharana
$\square$ Anguish, Stress, anxiety
$\square$ Indiscipline life style and food habits
$\square$ Contaminated environment
$\square$ Insufficient nutrition
$\square$ Infectious predominance
$\square$ Diminished Agni status
$\square$ Disease condition enfeebling Agni
$\square$ Virudha-Ahara

Ignorance regarding consideration of Desha and Kala during food consumption

\section{Signs and Symptoms}

The patient passes stool in large quantity either in solid or liquid form frequently along with symptoms like Trishna (Thirst), Aruchi (Anorexia), Virasya (Distaste of Mouth), Praseka (Excessive salivation). He also suffers from oedema in 
Legs and Hands, Pain in Bones and Phalanges, Chardi (Vomiting), Jwara (Fever) and Tiktha Amalodhgaara (Eructation having metabolic smell of Ama and Bitter as well as sour taste $)^{15}$. As per Acharaya Sushruta, the patient afflicted with Grahani Roga grumbles of Oedema over hands and feet, debility, pain in the little joints of hands and feet, greediness for food, thirst, retching, fever, anorexia and burning sensation ${ }^{16}$.

\section{LIST OF RASAUSHADHIS FOR GRAHANI ROGA MENTIONED IN BHAISHAJYA RATNAVALI}

\begin{tabular}{|c|c|c|c|}
\hline \multirow{2}{*}{ Sl.No } & \multirow{2}{*}{ Name } & \multicolumn{2}{|c|}{ Ingredients } \\
\hline & & Rasa Dravyas & Other ingredients \\
\hline 1 & Madhyama Gangadhara Churna & $\begin{array}{l}\text { Shuddha Parada and Shuddha } \\
\text { Gandhaka }\end{array}$ & $\begin{array}{l}11 \text { drugs - Bilwa, } \\
\text { Mocharasa, Patha etc., }\end{array}$ \\
\hline 2 & Brihatlavangadya Churna & $\begin{array}{l}\text { Abhraka Bhasma, Loha Bhasma, } \\
\text { Shuddha Parada and Shuddha } \\
\text { Gandhaka }\end{array}$ & $\begin{array}{l}42 \text { drugs - Lavanga, } \\
\text { Ativisha, Musta etc., }\end{array}$ \\
\hline 3 & Lavangaadhyam Churna & $\begin{array}{l}\text { Abhraka bhasma, Loha bhasma, } \\
\text { Shuddha Parada and Shuddha } \\
\text { Gandhaka }\end{array}$ & $\begin{array}{l}39 \text { drugs - Lavanga, } \\
\text { Jeeraka, Harenuka etc., }\end{array}$ \\
\hline 4 & Swalpanaayika Churna & $\begin{array}{l}\text { Shuddha Parada and Shuddha } \\
\text { Gandhaka }\end{array}$ & $\begin{array}{l}8 \text { drugs }- \text { Pancha } \\
\text { Lavana, } \\
\text { Shuddha Vijaya } \text { etc., }\end{array}$ \\
\hline 5 & Madhyamanaayika Churna & $\begin{array}{l}\text { Abhraka Bhasma, Loha Bhasma, } \\
\text { Shuddha Parada and Shuddha } \\
\text { Gandhaka }\end{array}$ & $\begin{array}{l}\text { drugs }- \text { Pancha } \\
\text { Lavana, } \quad \text { Trikatu, } \\
\text { Shuddha Vijaya } \text { etc., }\end{array}$ \\
\hline 6 & Brihanaayika churna & $\begin{array}{l}\text { Abhraka Bhasma, Shuddha } \\
\text { Tankana, Shuddha Parada and } \\
\text { Shuddha Gandhaka }\end{array}$ & $\begin{array}{l}27 \text { drugs - Chitraka, } \\
\text { Triphala, Vyosha etc., }\end{array}$ \\
\hline 7 & Grahanishaardoola Churna & $\begin{array}{l}\text { Abhraka Bhasma, Loha Bhasma, } \\
\text { Shuddha Parada and Shuddha } \\
\text { Gandhaka }\end{array}$ & $\begin{array}{l}26 \text { drugs }- \text { Hingu, } \\
\text { Pancha Lavana, Haridra } \\
\text { etc., }\end{array}$ \\
\hline 8 & Jeerakadya Churna & $\begin{array}{l}\text { Abhraka Bhasma, Shuddha } \\
\text { Parada and Shuddha Gandhaka, } \\
\text { Shuddha Tankana, }\end{array}$ & $\begin{array}{l}20 \text { drugs - Jeeraka, } \\
\text { Musta, Patha etc., }\end{array}$ \\
\hline 9 & Markandeya Churna & $\begin{array}{l}\text { Shuddha Tankana, Abhraka } \\
\text { Bhasma, Shuddha Parada, } \\
\text { Shuddha Hingula and Shuddha } \\
\text { Gandhaka }\end{array}$ & 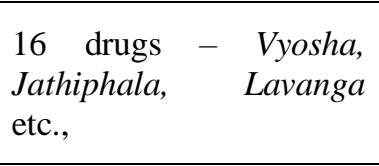 \\
\hline 10 & Shreekamaeshwara Modaka & Abhraka Bhasma & $\begin{array}{l}43 \text { drugs - Katphala, } \\
\text { Kushta, Ashwagandha } \\
\text { etc., }\end{array}$ \\
\hline 11 & Jeerakaadi Modaka & $\begin{array}{l}\text { Abhraka Bhasma, Loha Bhasma, } \\
\text { Vanga Bhasma }\end{array}$ & $\begin{array}{l}41 \text { drugs }- \text { Jeeraka, } \\
\text { Vijaya } \\
\text { Talisapatra etc., }\end{array}$ \\
\hline 12 & Brihat Jeerakaadi Modaka & $\begin{array}{l}\text { Abhraka Bhasma, Loha Bhasma, } \\
\text { Vanga Bhasma }\end{array}$ & $\begin{array}{l}48 \text { drugs }- \text { Jeeraka, } \\
\text { Kushta, Shunti etc., }\end{array}$ \\
\hline 13 & Agnikumara Modaka & Abhraka Bhasma, Loha Bhasma, & $\begin{array}{l}34 \text { drugs - Usheera, } \\
\text { Netrabaala, Musta etc., }\end{array}$ \\
\hline 14 & Hamsapottali Rasa & $\begin{array}{l}\text { Kaparda Bhasma, Shuddha } \\
\text { Gandhaka, Shuddha Parada }\end{array}$ & $\begin{array}{l}5 \text { drugs }- \text { Trikatu, } \\
\text { Visha,Jambeera etc., }\end{array}$ \\
\hline 15 & Grahanikaparda Pottali Rasa & $\begin{array}{l}\text { Kaparda Bhasma, Shuddha } \\
\text { Tankana, Shuddha Gandhaka, } \\
\text { Shuddha Parada }\end{array}$ & $1 \mathrm{drug}-$ Vijaya \\
\hline 16 & Agnikumara Rasa & $\begin{array}{l}\text { Shuddha Gandhaka, Shuddha } \\
\text { Parada, Shuddha Tankana, } \\
\text { Abhraka Bhasma, Loha Bhasma, }\end{array}$ & $\begin{array}{l}7 \text { drugs - Vatsanabha, } \\
\text { Trikatu, Ajamoda etc., }\end{array}$ \\
\hline 17 & Grahanikapata Rasa (Swalpa) & $\begin{array}{l}\text { Shuddha Hingula, Shuddha } \\
\text { Gandhaka, Varatika Bhasma }\end{array}$ & $\begin{array}{l}3 \quad \text { drugs } \\
\text { Vamshalochana, } \\
\text { Ahiphena, Aja Ksheera }\end{array}$ \\
\hline
\end{tabular}




\begin{tabular}{|c|c|c|c|}
\hline 18 & Grahanikapata Rasa (Dwitiya) & $\begin{array}{l}\text { Shuddha Parada, Shuddha } \\
\text { Gandhaka }\end{array}$ & 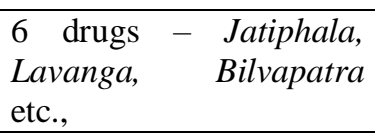 \\
\hline 19 & Grahanikapata Rasa (Tritiya) & $\begin{array}{l}\text { Shuddha Parada, Shuddha } \\
\text { Gandhaka }\end{array}$ & $\begin{array}{l}4 \text { drugs - Shwetasarja, } \\
\text { Suryavarta, Bilwa, } \\
\text { Shringataka }\end{array}$ \\
\hline 20 & Grahanikapata Rasa (Chaturtha) & $\begin{array}{lll}\text { Shuddha } & \text { Parada, } & \text { Shuddha } \\
\text { Gandhaka }\end{array}$ & $\begin{array}{l}2 \text { drugs }- \text { Ardraka, } \\
\text { Kutaja }\end{array}$ \\
\hline 21 & Grahanikapata rasa (Panchama) & $\begin{array}{l}\text { Shuddha Parada, Shuddha } \\
\text { Gandhaka, Shuddha Tankana, }\end{array}$ & $\begin{array}{l}12 \text { drugs - Jatipahala, } \\
\text { Bilwa, Khadira etc., }\end{array}$ \\
\hline 22 & Grahanivajrakapata Rasa & $\begin{array}{l}\text { Shuddha Parada, Shuddha } \\
\text { Gandhaka, Abhraka Bhasma, }\end{array}$ & $\begin{array}{l}9 \text { drugs }- \text { Jayanti, } \\
\text { Vacha, Bhringaraja etc., }\end{array}$ \\
\hline 23 & Grahanivajrakapata Rasa (Brihat) & $\begin{array}{l}\text { Rajata Bhasma, Mukta Bhasma, } \\
\text { Swarna Bhasma, Loha Bhasma, } \\
\text { Shuddha Parada, Shuddha } \\
\text { Gandhaka }\end{array}$ & $\begin{array}{l}9 \text { drugs - Khadira, Bala, } \\
\text { Apamarga etc., }\end{array}$ \\
\hline 24 & Sangrahagrahanikapata Rasa & $\begin{array}{l}\text { Mukta Bhasma, Swarna Bhasma, } \\
\text { Shuddha Tankana, Shuddha } \\
\text { Parada, Shuddha Gandhaka, } \\
\text { Abhraka Bhasma, Kaparda } \\
\text { Bhasma, Shankha Bhasma }\end{array}$ & $\begin{array}{l}5 \quad \text { drugs } \\
\text { Vatsanabha,Ativisha, } \\
\text { Vijaya, Chitraka, Musali }\end{array}$ \\
\hline 25 & Grahanigajendra Vatika & $\begin{array}{l}\text { Shuddha Tankana, Shuddha } \\
\text { Parada, Shuddha Gandhaka, } \\
\text { Loha bhasma, Shankha Bhasma }\end{array}$ & $\begin{array}{l}22 \text { drugs - Hingu, Shati, } \\
\text { Taleesapatra etc., }\end{array}$ \\
\hline 26 & Grahanishaardula Rasa & $\begin{array}{l}\text { Swarna Bhasma, Shuddha } \\
\text { Parada, Shuddha Gandhaka }\end{array}$ & $\begin{array}{l}5 \text { drugs }- \text { Lavanga, } \\
\text { Nimbapatra, Jatikosha, } \\
\text { Jatiphala, Sukshma Ela }\end{array}$ \\
\hline 27 & Vajrakapato Rasa & $\begin{array}{l}\text { Shuddha Parada, Shuddha } \\
\text { Gandhaka }\end{array}$ & $\begin{array}{lr}10 \text { drugs }- & \text { Ahiphena, } \\
\text { Mocharasa, } & \text { Trikatu, } \\
\text { Triphala, } & \text { Vijaya, } \\
\text { Bhringaraja } & \end{array}$ \\
\hline 28 & Mahagandhakam & $\begin{array}{l}\text { Shuddha Parada, Shuddha } \\
\text { Gandhaka }\end{array}$ & $\begin{array}{l}6 \text { drugs }- \text { Jatiphala, } \\
\text { Jatikosha, } \\
\text { Nimbapatra, } \\
\text { Sindhuvara, Ela }\end{array}$ \\
\hline 29 & Shreevaidyanatha Vatika & $\begin{array}{l}\text { Shuddha Parada, Shuddha } \\
\text { Gandhaka }\end{array}$ & $\begin{array}{l}16 \text { drugs }- \text { Chitraka, } \\
\text { Triphala, Bhringaraja } \\
\text { etc., }\end{array}$ \\
\hline 30 & Khasarpana Vatika & $\begin{array}{l}\text { Shuddha Parada, Shuddha } \\
\text { Gandhaka }\end{array}$ & $\begin{array}{l}9 \text { drugs }- \text { Haridra, } \\
\text { Bhringaraja, } \\
\text { Mandukaparni etc., }\end{array}$ \\
\hline 31 & Rasabhra Vati & $\begin{array}{ll}\text { Shuddha Parada, } & \text { Shuddha } \\
\text { Gandhaka, Abhraka } & \text { Bhasma, } \\
\text { Shuddha Tankana } & \\
\end{array}$ & $\begin{array}{l}12 \text { drugs - } \text { Kesharaja, } \\
\text { Bhringaraja, Nirgundi } \\
\text { etc., }\end{array}$ \\
\hline 32 & Mahabhra Vati & $\begin{array}{l}\text { Abhraka Bhasma, Loha Bhasma, } \\
\text { Tamra Bhasma, Shuddha } \\
\text { Manahshila, Shuddha Tankana, } \\
\text { Shuddha Parada and Shuddha } \\
\text { Gandhaka }\end{array}$ & $\begin{array}{l}25 \text { drugs }- \text { Krishnasarpa } \\
\text { Visha, Triphala, } \\
\text { Bhringaraja etc., }\end{array}$ \\
\hline 33 & Peeyushavalli Rasa & $\begin{array}{l}\text { Abhraka Bhasma, Loha } \\
\text { Bhasma,Rajata Bhasma, Tamra } \\
\text { Bhasma Shuddha Parada and } \\
\text { Shuddha Gandhaka, Swarna } \\
\text { Makshika Bhasma }\end{array}$ & $\begin{array}{l}19 \text { drugs - Lavanga, } \\
\text { Shweta } \\
\text { Patha etc., }\end{array}$ \\
\hline 34 & Paneeyabhakta Vati & Abhraka Bhasma, Loha Bhasma & $\begin{array}{l}19 \text { drugs - Vayuvidanga, } \\
\text { Chavya, Shunti etc., }\end{array}$ \\
\hline 35 & Shreenrupathivallabha Rasa & $\begin{array}{l}\text { Abhraka Bhasma, Loha Bhasma, } \\
\text { Tamra Bhasma Shuddha Parada }\end{array}$ & $\begin{array}{l}14 \text { drugs -Jatiphala, } \\
\text { Lavanga, Musta etc., }\end{array}$ \\
\hline
\end{tabular}




\begin{tabular}{|c|c|c|c|}
\hline & & and Shuddha Gandhaka & \\
\hline 36 & Brihat Nrupavallabha Rasa & $\begin{array}{l}\text { Abhraka Bhasma, Loha Bhasma, } \\
\text { Naga Bhasma Shuddha Parada } \\
\text { and Shuddha Gandhaka, } \\
\text { Shuddha Tankana, Rajata } \\
\text { Bhasma, Swarna Bhasma }\end{array}$ & $\begin{array}{l}15 \text { drugs - Chitraka, } \\
\text { Nishotha, Jatiphala etc., }\end{array}$ \\
\hline 37 & Maharaja Nrupathivallabha Rasa & $\begin{array}{l}\text { Abhrak Bhasma, Loha Bhasma, } \\
\text { Tamra Bhasma Shuddha Parada } \\
\text { and Shuddha Gandhaka, Rajata } \\
\text { Bhasma, Swarna Bhasma, } \\
\text { Swarna Makshika Bhasma, } \\
\text { Shuddha Tankana }\end{array}$ & $\begin{array}{l}25 \text { drugs - Bhringaraja, } \\
\text { Gajapippalai, Danti etc., }\end{array}$ \\
\hline 38 & Maharaja Nrupavallabha Rasa & $\begin{array}{l}\text { Abhraka Bhasma, Loha Bhasma, } \\
\text { Tamra Bhasma, Shuddha } \\
\text { Gandhaka, Rajata Bhasma, } \\
\text { Swarna Bhasma, Swarna } \\
\text { Makshika Bhasma, Vanga } \\
\text { Bhasma, ShankhaBhasma }\end{array}$ & $\begin{array}{l}19 \text { drugs - Pippalimula, } \\
\text { Shunti, } \quad \text { Saindhava } \\
\text { Lavana etc., }\end{array}$ \\
\hline 39 & Jaatipalaadi Vati & $\begin{array}{l}\text { Shuddha Tankana, Abhraka } \\
\text { Bhasma }\end{array}$ & $\begin{array}{l}4 \text { drugs }- \text { Jatiphala, } \\
\text { Vijaya, } \\
\text { Gandhaprasarini }\end{array}$ \\
\hline 40 & Jaatipalaadya Vati & 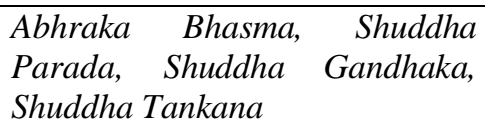 & $\begin{array}{l}19 \text { drugs - Jatiphala, } \\
\text { Mocharasa, Musta etc., }\end{array}$ \\
\hline 41 & Vadavaamukha Rasa & $\begin{array}{lcl}\text { Abhraka } & \text { Bhasma, } & \text { Shuddha } \\
\text { Parada, } & \text { Tamra } & \text { Bhasma, } \\
\text { Shuddha } & \text { Gandhaka, } & \text { Shuddha } \\
\text { Tankana } & & \\
\end{array}$ & $\begin{array}{l}10 \text { drugs }- \text { Samudra } \\
\text { Lavana, Yavakshara, } \\
\text { Sarjika Kshara etc., }\end{array}$ \\
\hline 42 & Rasa Parpati & $\begin{array}{l}\text { Shuddha Parada, } \quad \text { Shuddha } \\
\text { Gandhaka }\end{array}$ & $\begin{array}{l}7 \text { drugs }- \text { Bhringaraja } \\
\text { Triphala, ghritakumari } \\
\text { etc., }\end{array}$ \\
\hline 43 & Loha Parpati & $\begin{array}{l}\text { Shuddha Parada, Shuddha } \\
\text { Gandhaka, Loha Bhasma }\end{array}$ & - \\
\hline 44 & Swarna Parpati & $\begin{array}{l}\text { Shuddha Parada, Shuddha } \\
\text { Gandhaka, Swarna Bhasma }\end{array}$ & - \\
\hline 45 & Panchamrita Parpati & $\begin{array}{l}\text { Abhraka Bhasma, Loha Bhasma, } \\
\text { Tamra Bhasma, Shuddha } \\
\text { Gandhaka, Shuddha Parada }\end{array}$ & - \\
\hline 46 & Vijaya Parpati & $\begin{array}{l}\text { Shuddha Gandhaka, Shuddha } \\
\text { Parada, Rajata Bhasma, Swarna } \\
\text { Bhasma, Vajra Bhasma, Muktha } \\
\text { Bhasma }\end{array}$ & 1 drug - Bhringaraja \\
\hline 47 & Hiranyagarbha Pottali Rasa & $\begin{array}{llr}\text { Shuddha } & \text { Parada, } & \text { Swarna } \\
\text { Bhasma, } & \text { Mukta } & \text { Bhasma, } \\
\text { Shankha } & \text { Bhasma, } & \text { Shuddha } \\
\text { Gandhaka, Kapardika } & \text { Bhasma, } \\
\text { Shuddha Tankana } & \end{array}$ & 1 drug - Nimbu Swarasa \\
\hline 48 & Rasendra Churnam & $\begin{array}{l}\text { Rasasindhura, Muktha Bhasma, } \\
\text { Swarna Bhasma }\end{array}$ & $\begin{array}{l}3 \quad \text { drugs } \\
\text { Vamshalochana, } \\
\text { Ahiphena, Goksheera }\end{array}$ \\
\hline 49 & Purnakala Vati & $\begin{array}{l}\text { Shuddha Gandhaka, Shuddha } \\
\text { Parada, Loha Bhasma, Shuddha } \\
\text { Tankana, Abhraka Bhasma }\end{array}$ & $\begin{array}{l}26 \text { drugs }- \text { Musta, } \\
\text { Dhataki Pushpa, Bilwa } \\
\text { etc., }\end{array}$ \\
\hline 50 & Shambhukadi Vati & Shambuka Bhasma & $\begin{array}{l}2 \text { drugs - Saindhava } \\
\text { Lavana,Madhu }\end{array}$ \\
\hline 51 & Agastyasootaraja Rasa & $\begin{array}{l}\text { Shuddha Gandhaka, Shuddha } \\
\text { Parada, Shuddha Hingula }\end{array}$ & $\begin{array}{l}3 \text { drugs - Ahiphena, } \\
\text { Dustura, Bhringaraja }\end{array}$ \\
\hline 52 & Agnisunu Rasa & Kapardika Bhasma, Shankha & 2 drugs $-\mathrm{Nimbu}$ \\
\hline
\end{tabular}




\begin{tabular}{|c|l|l|l|}
\hline & & $\begin{array}{l}\text { Bhasma, Shuddha Gandhaka, } \\
\text { Shuddha Parada }\end{array}$ & Swarasa, Maricha \\
\hline 53 & Dugdha Vati & $\begin{array}{l}\text { Shuddha Hingula } \\
\text { 6 drugs - Devapushpa, } \\
\text { Ahiphena, Visha etc., }\end{array}$ \\
\hline 54 & Dugdha Vati Dwitiya & $\begin{array}{l}\text { Kantaloha Bhasma, Abhraka } \\
\text { Bhasma drugs - Vatsanabha, } \\
\text { Ahiphena, Goksheera }\end{array}$ \\
\hline 55 & Panchamrita Mandura & $\begin{array}{l}\text { Loha Bhasma, Tamra Bhasma, } \\
\text { Shuddha Gandhaka, Shuddha } \\
\text { Parada, Abhraka Bhasma, } \\
\text { Shuddha Mandura }\end{array}$ & $\begin{array}{l}22 \text { drugs } \\
\text { Trikatu,Triphala,Musta, } \\
\text { Vayuvidanga etc., }\end{array}$ \\
\hline
\end{tabular}

\section{DISCUSSION}

The Ayurvedic Science proposes that Grahani Roga can be treated by adopting the idea of Langhana, Deepana-Pachana using suitable medications that enhance the Agni and removes the Ama. Acharya Bhava Praksha says that Grahani Dosha ought to be dealt with like that of Ajeerna Roga. He also specifies the usage of Atisarahara Dravyas along with Deepaniya Dravyas and Langhana ${ }^{17}$.

Although many herbal formulations have been mentioned for the Chikitsa(treatment) of Grahani Roga, Rasaushadhis play a major part because of Alpamaatropayogitvat(requirement of very small dose), kshipramarogyadhayitvat(instant effectiveness) and Yogavahi(synergistic) property ${ }^{18}$.

Here, Sulphur acts by its Ushna Virya, Tikshna, Yogavahi, Amadoshahara properties and Rasayana, Sheeta Shamana, Deepana, Brimhana, Balya functions mitigate Vata Kapaha Dosha, helps in bringing back Agni to normalcy and thereby correcting Ajeerna. Sulphur is present in numerous biological molecules like cysteine, taurine, cystin, methionine and glutathione etc., antioxidant enzymes ${ }^{19}$. These biomolecules serve as curing factors in Ajeerna whereas Parada acts as transitory catalyst $\mathrm{t}^{20}$.

Deepana Pachana property of Kaparda Bhasma helps to relive deranged Pitta. As per modern science, Kaparda Bhasma contains calcium carbonate. Calcium has a unique role in cell physiology in living organisms. It takes part in the production of many enzymes and hormones which regulate the digestion process and metabolism ${ }^{21}$.

Bhasmas and Rasa dravyas like Abhraka Bhasma, Tamra Bhasma, Vanga Bhasma, Kaparda Bhasma, Mukta Bhasma, Shankha Bhasma, Naga Bhasma, Rasasindhura, Mandura Bhasma, Swarnabhasma, Rajatabhasma, Swarnamakshikabhasma, Shuddha Tankana are having Deepana action and hence indicated in Grahani Roga.

Shuddha Hingula and Shambuka Bhasma are having both Deepana and Pachana action. Tamra, Kaparda, Shankha, Naga are specifically indicated for Grahani Roga. Shankha Bhasma, Shambuka Bhasma and Gandhaka have Udara Shoolahara property.

Parada, Swarnamakshika, Vajra are having Yogavahi property thereby potentiating the herbal drugs having the properties like stool bulking agents, anti-spasmodics, anti-diarrheals, antiflatulents, modulation of gut flora.

Grahani Roga is chiefly caused by Mandagni. Henceforth rebuilding of Agni is the core area of concern in the treatment of Grahani Roga. Parpati preparation involves Agni Samskara through which appetite is improved due to Deepana and Ushna properties of Agni. As Vata gets alleviated the symptoms like Atipravritti of Mala switch to Samyak 
Pravritti. Poshaka Rasa gets absorbed into the Srotas as the intestinal ducts are opened by the Deepana, Pachana and Ushna Gunas of the Dravyas.

The metals and minerals present in these Yogas aid in mitigating the Vata, Pitta and Kapha Doshas as they have attributes like Kashaya Rasa, Ruksha Guna, Ushna Veerya, Madhura Vipaka and thereby potentiating the Agni which ameliorates the digestion process and helps in the management of a disease.

\section{CONCLUSIONS}

Grahani Roga is a Annavaha Srotovyadhi concerned with Agni and a way of living. As per Ayurveda impairment of Jatharagni, Samana Vayu, Pachaka Pitta and Kledaka Kapha are responsible for the manifestation of Grahani Roga hence regarded as Tridoshatmaka Vyadhi. This illness is portrayed by Stomach torments, distended abdomen and disturbed bowel habits. In classics, discrete dosage forms have been mentioned for the management of Grahani Roga among which Rasaushadhis have supremacy due to their swift action with minimal dose. By utilization of these medications as a solitary medication or in combination one can handle as well as deal with the illness very well.

\section{REFERENCES}

1. Tripathi Brahmanand and Pandey G.S, Charaka Samhita of Agnivesa with Charaka Chandrika Hindi Commentory, 6th Ed., Varanasi: Chaukhamba Surbharati Prakashan., (Vol-1), 1999.

2. Shastri K. Ambikadatta, Sushruta Samhita of Maharsi Sushrut edited with Ayurveda Tattva Sandipika Hindi Commentary, 13th Ed., Varanasi: Chaukhamba Sanskrit Bhawan., (Vol-2), 2000.

3. Vridda Jeevaka- Kashyapa Samhita (Vriddajeevaka Tantra) by -Sharma Hemaraj Pandit (Nepal Rajguru) revised by Vatsya with Sanskrit introduction and Vidyothini Hindi Commentary, Varanasi: Chaukhamba Sanskrit Sansthan., 1994.

4. Rama Valamba Shashtri, Harita Samhita Edited with Asha Hindi Commentary, Varanasi: Prachya Prakashan., 1985.

5. Longo, Fauci,Kasper, Hauser, Jameson, Loscalizo. Harrison's Principle of Internal Medicine, 18 ed. United States of America: McGraw Hill, 2012

6. 6 ) Sharma Dr.Ram Karan and Dash Vaidya Bhagawan, Charaka Samhita of Agnivesha, 2 ed. Varanasi, Chaukhamba Sanskrit Series Office,2000, Chikitsa Sthana 15/3-4, p- 1

7. Sharma Dr.Ram Karan and Dash Vaidya Bhagawan, Charaka Samhita of Agnivesha, 2 ed. Varanasi, Chaukhamba Sanskrit Series Office,2000, Chikitsa Sthana 15/ 51-54, p- 28

8. Sharma Dr.Ram Karan and Dash Vaidya Bhagawan, Charaka Samhita of Agnivesha, 2 ed. Varanasi, Chaukhamba Sanskrit Series Office,2000, Chikitsa Sthana 15/56-57, p-29

9. Shastri Kaviraja Ambikadutta, Susruta Samhita of Maharishi Sushruta, Varanasi, Chaukhamba Sanskriti Sansthan, 2008, Uttaratantra 40/169, p- 237.

10. Gupta Kaviraj Atrideva, Ashtanga Samgraha, Varanasi, ChawkhambaKrishnadas Academy, Sharir Sthana 5/23, p- 299.

11. Vaidya Yadavaji Trikamji. Charaka Samhita with Ayurveda Dipika Commentary of Chakrapanidatta, Varanasi, Chaukhambha Prakashan, Chikitsa Sthana 15/12, p- 513.

12. Shastri Pt. Kashinatha and Chaturvedi Dr Gorakhanatha, Charaka Samhita of Agnivesha, Varanasi, Chaukhamba Bharti Academy, 2007, Chikitsa Sthana 15/44, p- 460.

13. Shastri Kaviraja Ambikadutta, Susruta Samhita of Maharishi Sushruta, Varanasi, Chaukhamba Sanskriti Sansthan, 2008, 
Uttaratantra 40/167, p- 237.

14. Jain, S., Chawardol, S., Jain, J. and Dwivedi, O., 2018. Management of Grahani Roga by Ayurveda principles and life style modification. Journal of Drug Delivery and Therapeutics, 8(6), pp.393-396.

15. Shastri Pt. Kashinatha and Chaturvedi Dr Gorakhanatha, Charaka Samhita of Agnivesha, Varanasi, Chaukhamba Bharti Academy, 2007, Chikitsa Sthana 15/51-54, p- 461.

16. Shastri Kaviraja Ambikadutta, Susruta Samhita of Maharishi Sushruta, Varanasi, Chaukhamba Sanskriti Sansthan, 2008, Uttaratantra 40/173-174, p- 239.

17. Brahma Shankara Mishra and Rupalalji Vaishya. Bhavprakasha of Shri Bhava Mishra, Part I (Madhya Khanda, Grahani Adhikara, Verse 27). Varanasi; Chaukhambha Sanskrit Bhawan; 1990. p. 34

18. Tripathi Indradeva. Rasendra Sara Sangraha. Varanasi: ChaukhambhaOrientalia; $2^{\text {nd }}$ ed. 1998.

19. Brosnan JT et al: The sulfur-containing amino acids: an overview. J Nutr. 2006;136(6 Suppl):p. 1636-1640.

20. Chun W. Lee, Shannon D. Serre, Yongxin Zhao, Sung Jun Lee \& Thomas W. Hastings (2008) Mercury Oxidation Promoted by a Selective Catalytic Reduction Catalyst under Simulated Powder River Basin Coal Combustion Conditions, Journal of the Air \& Waste Management Association, 58:4, 484-493.

21. Piste Pravina, Didwagh Sayaji and Mokashi Avinash (2013) Calcium and its Role in Human Body. International Journal of Research in Pharmaceutical and Biomedical Sciences; 4 (2):659- 668.

22. MASIH, JOLLY, and AMITA SHARMA. "STUDY ON MARKETING POTENTIAL FOR CHANNELS INVOLVED IN MARKETING OF GLUTEN-FREE FOODS IN INDIA AND USA." International Journal of Agricultural Science and Research (IJASR) 8.4, Aug 2018, 111-120

23. TAHSIN, MD HANIF. "MEDICINAL PLANTS USED BY TRADITIONAL HEALERS FOR SKIN DISEASES AT PIRGANJ IN BANGLADESH." International Journal of Biological Research and Development (IJBRD) 11.1, Jun 2021, 25-48

24. HAMEED, ALIR, Luma T. Ahmed, and Sabah M. Ali. "Molecular and Genotypes Identification of C. Albicans Isolated from Children With Diarrea in Diyala Province-Iraq. "International Journal of Bio-Technology and Research (IJBTR) 7.6: 1-10.

25. Al-Saffar, Anwar Kadhim. "The Effect of Mixture (Sidr Extract with Hydrogen Peroxide) in the Disinfection of Hospital Instrument." International Journal of Botany and Research (IJBR) 4.3: 7-10. 